\title{
Editorial
}

\section{What have we learnt from AIDS?}

What have we learnt from AIDS? Or perhaps, what have we not learnt from AIDS? Because the issues raised by AIDS have reached into virtually every part of the biological sciences, medicine, and society, the opportunity to re-evaluate our preconceptions and our practice has been universal. To what extent have we risen to the sometimes uncomfortable challenges AIDS has brought? Perhaps it is best to throw the question back to you the reader: in what ways do you think and behave differently from 16 years ago as a result of AIDS?

The impact of AIDS has been as pervasive as any infection in history - tuberculosis, leprosy, and syphilis being obvious forbears. It has forced us to re-examine perceptions about the containment of microbial threats, even though many other infections have been there to remind us. This emphasises that none of the issues raised by AIDS are unique or truly new. Rather, because AIDS was new and engaged our attention, it has obliged us to re-examine issues that have always surrounded human pathology, although they have not always been fully addressed. The relevance of previous solutions, whether they were adequate or appropriate, had to be re-assessed.

Similarly, the lessons learnt are not and cannot be unique to AIDS. Sadly, the perception exists of a wish to preserve a spurious separateness for AIDS issues. What we learn from AIDS must be applicable to other diseases. If the lessons are not exportable or translatable, either they are not relevant or, more likely, we are denying their relevance.

AIDS has enormously enhanced our understanding of the host-parasite relation, not only with HIV itself but also with a wide range of pathogens that cause opportunist infections and tumours. An organism that targets key cells of the immune system has illuminated the fine workings of these defences. Fortunately, many tools for identifying cell surface markers, cytokines, and intracellular signalling pathways have become available simultaneously, ensuring that many of the hypotheses are testable. Better insight into immune regulation and its differentiated function in health and disease has come from the worked example of HIV pathogenesis. Similarly, we are learning more about host-parasite interactions for organisms as diverse as candida, pneumocystis, mycobacteria, leishmania, and herpesviruses, including the newly identified $\mathrm{HHV}-8$, and the pathogenesis of Kaposi's sarcoma.

Recent concerns about the extent to which AIDS research has been funded seem to be curiously narrow turf disputes that ignore the central relevance of the concepts unveiled by AIDS. The application of resources to new diseases, whether rare or (as in this case) common, has usually fostered new insights of wider relevance. The global scale of the pandemic, much of it focused in the developing world, surely demands a substantial response from research in the industrialised world. It need not detract from work on other key health issues such as malaria and tuberculosis, and indeed often informs these directly. We should see the approach as being "both/and" not "either/or".
Issues of infection control and risk assessment have been re-opened by HIV - whether at the level of the individual in regard to sexual, drug using, and childbearing behaviours, or the population in terms of public policy, blood transfusion, and health care. We have been obliged to understand better the extent to which these are accessible to personal or social behaviour modification or to the regulation of clinical practice and procedures. Health care workers infected by HIV or other bloodborne pathogens have presented some particularly difficult questions. Overall, we are still coming to terms with the reality that we cannot control all conceivable or theoretically preventable risks. Rather we need to identify realistic and sustainable interventions.

Appropriate and affordable health care for people with HIV in industrialised and developing countries has been imaginatively shaped by clinicians from many backgrounds, forging contemporary models of care that are now changing the way we address the needs of other care groups. This has been enhanced by the enthusiastic advocacy of affected people, suggesting new approaches to partnership in care.

The recent emergence of combination antiretroviral therapies has offered tremendous opportunities for improving prognosis, but also exposes tensions in resource allocation. How can we find the wherewithal to invest in these treatments to allow lower long term health care costs and increased economic productivity of those affected? The issue of emerging and initially expensive treatments is reminiscent of early advances in the treatment of leukaemia and renal failure, although it is now in the context of more overtly strained health economies; emerging treatments for other common disorders will soon pose similar problems. Affordable solutions for the developing world, whether through treatment or potential prophylaxis, remind us of the limited access to hepatitis $B$ vaccines in the regions most affected, and many other resource limitations in global public health or disease management.

Development of new drugs through clinical trials has been critically scrutinised, not only by clinicians and scientists but also by those affected. It has been hard to balance the immediate needs of current patients with obtaining robust data to inform future treatment. While some shortcuts have been successful, in other instances we have been forcibly reminded why substantial controlled studies are essential. Clinicians and patients still need to cope with uncertainty and to avoid raising unrealisable expectations, exemplified by the perhaps excessive optimism about achievable goals with current combination therapy.

AIDS has re-opened ethical debates about consent, confidentiality (due to the social stigma of the disease and of the groups affected), and perceived or actual tensions between individual rights and public health. Policy has had to be developed when information is necessarily incomplete and where media and other pressures encourage irrational or unjustifiable responses. It has required considerable maturity of scientific advice, public debate, and political response. More recent debates on BSE and new variant Creutzfeldt-Jakob disease have shown how widely 
applicable are the lessons and where these have not yet been fully learnt.

The personal and social lessons of AIDS remain incompletely learnt. While stigma and misunderstanding have reduced, substantial problems remain. The reduced media and political interest in AIDS are a mixed blessing. Some issues can usefully be resolved away from the spotlight but there is the ever present danger that uncomfortable or recalcitrant problems will now be swept back under the carpet. It remains for us to produce durable and, above all, generalisable responses.

\section{ANTHONY J PINCHING}

Louis Freedman Professor of Immunology,

St Bartholomew's and the Royal London School

of Medicine and Dentistry,

Queen Mary and Westfield College, London, UK 